\title{
Gestión de la recaudación tributaria en las municipalidades del Perú
}

\author{
Flor Marina Rojas Benites \\ florrojas83@gmail.com \\ ORCID: 0000-0002-2869-6666 \\ Escuela de Posgrado \\ Universidad César Vallejo \\ Hipólito Percy Barbarán Mozo \\ pbarmozito@ hotmail.com \\ ORCID: 0000-0002-9316-202X \\ Escuela de Posgrado \\ Universidad César Vallejo
}

\section{RESUMEN}

La gestión de la recaudación está compuesta por diferentes acciones enfocadas a garantizar que la entidad obtenga el dinero necesario para solventar los compromisos $\mathrm{u}$ obligaciones, así como para prestar servicios públicos de calidad y mejorar las condiciones de vida de la población. El objetivo de la investigación fue identificar los principales nudos críticos en la gestión de la recaudación en las municipalidades del Perú durante los últimos años, bajo un enfoque descriptivo y cualitativo con diseño de revisión sistemática, con una muestra de 12 artículos científicos referidos a la gestión de la recaudación en las municipalidades, recogidos de Redalyc, Scielo y otras bases de datos, cuyos hallazgos caracterizan una problemática común, expresada en una serie de deficiencias administrativas y de gestión que han desencadenado en alta morosidad, evasión, desinformación, percepción negativa sobre la tributación, inconciencia tributaria, etc. Se concluye que, la gestión de la recaudación en las municipalidades del Perú durante los últimos años se ha agravado y presenta una serie de desatinos en el sistema administrativo tributario; por cuanto, no existen mecanismos efectivos para disciplinar la economía informal, la evasión tributaria y la corrupción, sumado la opinión imprecisa y negativa de los contribuyentes sobre los destinos de la recaudación, entre otros problemas que motiva la necesidad de edificar una propuesta de gestión más efectiva o un nuevo sistema tributario acorde con la realidad de cada municipio.

Palabras clave: gestión; recaudación; evasión; municipalidad. 


\title{
Management of tax collection in the municipalities of Peru
}

\begin{abstract}
Collection management is made up of different actions focused on ensuring that the entity obtains the money necessary to meet the commitments or obligations, as well as to provide quality public services and improve the living conditions of the population. The objective of the research was to identify the main critical nodes in the management of collection in the municipalities of Peru in recent years, under a descriptive and qualitative approach with a systematic review design, with a sample of 12 scientific articles referring to management of collection in the municipalities, collected from Redalyc, Scielo and other databases, whose findings characterize a common problem, expressed in a series of administrative and management deficiencies that have led to high delinquency, evasion, misinformation, negative perception about the taxation, tax unconsciousness, etc. It is concluded that the collection management in the municipalities of Peru in recent years has worsened and presents a series of blunders in the tax administrative system; as there are no effective mechanisms to discipline the informal economy, tax evasion and corruption, in addition to the imprecise and negative opinion of taxpayers on the destination of the collection, among other problems that motivate the need to build a more efficient management proposal. effective or a new tax system according to the reality of each municipality.
\end{abstract}

Keywords: management; collection; evasion; municipality.

Artículo recibido: 10 Setiembre. 2021 Aceptado para publicación: 15 Octubre. 2021

Correspondencia: florrojas83@gmail.com

Conflictos de Interés: Ninguna que declarar 


\section{INTRODUCCIÓN}

Desde una perspectiva internacional, se evidencia que diversos países otorgan ciertas atribuciones tales como la recaudación tributaria a los municipios como órganos del gobierno local. En tal sentido, Gonzáles y Mosquera (2020) indican que, en Colombia, esta atribución se encuentra regulada por el numeral 3 del Art. 267 de la Constitución Política y esto brinda autonomía para que los municipios gestionen y administren sus fondos en función a sus intereses, por lo que debe contar con los medios requeridos para la consecución efectiva de sus atribuciones. No obstante, a pesar de contar con este marco regulatorio y evidenciar un claro incremento de los importes de recaudación tributaria municipal, Pérez et al. (2019) mencionan que los ingresos obtenidos por los municipios colombianos sólo lograron representar un 2,4\% del PIB para el año 2017, siendo este mayor al 0,9\% del PIB que se obtuvo en el año 1990, donde Bucaramanga y Barranquilla son las áreas metropolitanas que tienen un mayor índice de recaudación tributaria municipal.

En Perú, la tributación de las municipales está regulada por el D.L. N776 y D.S. N¹562004-EF y es considerada al momento de llevar a cabo la elaboración del Presupuesto Institucional visto que los recursos municipales se originan principalmente por dos fuentes de financiación que son los recursos directamente recaudados, siendo estos obtenidos por el recaudo de tributos, y los recursos adquiridos por transferencia de los fondos públicos, por tanto, una mala gestión tributaria por parte de los municipios está asociada de forma directa con la falta de efectividad en las acciones de planeación y deficiente capacidad institucional, así como por el bajo nivel de cultura tributaria en la colectividad peruana (Morel et al., 2020).

El Ministerio de Economía y Finanzas (2019) informó que, para el año 2018, el 17\% de los ingresos que fueron obtenidos por las municipalidades corresponden a los tributos municipales, siendo esta cifra equivalente a S/ 4,425 millones, de los cuales el 64\% (S/ 2,826 millones) pertenecieron a los impuestos municipales entre los cuales destaca el impuesto predial y el 36\% (S/ 1,599 millones) pertenecieron a otros tributos municipales. De la misma manera, Melgarejo (2018) manifestó que el municipio de Lima pudo recaudar para el mes de octubre sólo S/ 16 millones por concepto de impuestos municipales, siendo este importe $74 \%$ menos de lo recaudado en el mes de setiembre y $56 \%$ menos en comparación con el mismo mes del año anterior; mientras que los distritos como San Borja 
y Miraflores han tenido una reducción del importe de recaudación de $75 \%$ y $70 \%$ respectivamente.

Igualmente, resulta conveniente destacar lo señalado por Redacción Andina (2020), por cuanto menciona que, con la finalidad de incentivar la recaudación de las municipalidades, el Estado ha creado el Plan de Incentivos que consiste en otorgar un importe económico a favor de un gobierno local como recompensa por la consecución oportuna de sus metas en base a su buen desempeño y eficiente gestión de sus recursos por lo que, para el presente periodo, se ha determinado que el MEF transfiere un suma equivalente a S/ 800 millones a las municipalidades que cumplieron sus metas propuestas.

Después de analizar esta problemática, se reconoce que la gestión de la recaudación debe ser considerada como una herramienta indispensable en la municipalidad por cuanto facilita la ejecución pertinente de los procesos de recaudo a fin de asegurar la obtención de los ingresos proyectados para un ejercicio fiscal. Es por ello que se considera propicio plantear como problema general: ¿Cuáles son los principales nudos críticos sobre la gestión de la recaudación tributaria en las municipalidades del Perú durante los últimos años?

\section{MATERIALES Y MÉTODOS}

El enfoque del estudio fue cualitativo, en vista de que Sánchez (2019) refiere que estos estudios emplean como parte de su proceso metodológico se emplean discursos, textos y documentos con la finalidad de facilitar la comprensión sobre ciertas cuestiones que permiten comprender la conducta de las variables.

De igual forma, el diseño de la investigación fue de revisión sistemática, dado que PardalRefoyo y Pardal-Peláez (2020) refieren que para el desarrollo de estas investigaciones se deben seguir procesos específicos al momento de recoger, analizar y detallar la información concerniente a un tema, evitando en todo momento presentar una cantidad significativa de limitaciones. Así también, presentó un diseño descriptivo donde Díaz-Narváez y Calzadilla (2016) aseveran que en los estudios que presentan este diseño se deben revisar conceptos, teorías y afirmaciones presentadas por autores que refieren sobre la gestión de la recaudación en los gobiernos locales con la finalidad de recoger la información científica necesaria para elaborar las conclusiones de acuerdo con el objetivo propuesto. 


\section{RESULTADOS Y DISCUSIÓN}

Mediante la revisión efectuada, se procede a presentar la siguiente tabla como parte de los resultados:

\begin{tabular}{|c|c|c|c|c|c|}
\hline $\mathbf{N}^{\circ}$ & Autor & País & Clasificación & Tipo y diseño & Conclusiones \\
\hline 1. & $\begin{array}{l}\text { Onofre, } \\
\text { Aguirre et } \\
\text { al. (2017) }\end{array}$ & Ecuador & $\begin{array}{l}\text { Artículo } \\
\text { científico }\end{array}$ & $\begin{array}{c}\text { Cuantitativo, } \\
\text { descriptivo }\end{array}$ & $\begin{array}{l}\text { La entidad presenta bajos índices de } \\
\text { recaudación debido a que el } 91 \% \text { no } \\
\text { está informado acerca del ente } \\
\text { responsable y los procesos que } \\
\text { deben seguir los contribuyentes al } \\
\text { momento de efectuar el pago del } \\
\text { impuesto. De igual forma, el } 42 \% \\
\text { afirma que la entidad es una mala } \\
\text { gestora de recaudación tributaria } \\
\text { debido a que no logra obtener el } \\
\text { importe de ingresos previstos en el } \\
\text { presupuesto, donde la ausencia de } \\
\text { una efectiva rendición de cuentas } \\
\text { impide que los contribuyentes } \\
\text { perciban el destino y uso eficiente } \\
\text { de los fondos obtenidos. }\end{array}$ \\
\hline 2. & $\begin{array}{l}\text { Camacho, } \\
\text { et al (2017) }\end{array}$ & Colombia & $\begin{array}{l}\text { Artículo } \\
\text { científico }\end{array}$ & $\begin{array}{l}\text { Cuantitativo } \\
\text { descriptivo }\end{array}$ & $\begin{array}{l}\text { El municipio desarrolla una gestión } \\
\text { de recaudación tributaria poco } \\
\text { eficiente debido a la presencia } \\
\text { significativas de factores tales como } \\
\text { la ausencia de documentos e } \\
\text { informes actualizados respecto al } \\
\text { recaudo tributario, el incremento de } \\
\text { los gastos administrativos y } \\
\text { operativos de la entidad, etc. Por } \\
\text { tanto, se requiere de forma urgente } \\
\text { la implementación de un sistema de } \\
\text { gestión que contenga procesos y } \\
\text { acciones acordes con el contexto } \\
\text { presentado. }\end{array}$ \\
\hline 3. & $\begin{array}{l}\text { Chávez y } \\
\text { López, } \\
\text { (2019) }\end{array}$ & México & $\begin{array}{l}\text { Artículo } \\
\text { científico }\end{array}$ & $\begin{array}{c}\text { Cuantitativo, } \\
\text { descriptivo }\end{array}$ & $\begin{array}{l}\text { El esfuerzo realizado por los } \\
\text { gobiernos locales de las zonas } \\
\text { rurales de la región no es suficiente } \\
\text { dado que en el período } 1989-2015 \\
\text { el índice de recaudación sólo ha } \\
\text { incrementado en un } 4.2 \% \text {, mientras } \\
\text { que los municipios en transición y } \\
\text { zonas urbanas obtuvieron índices de } \\
5.3 \% \text { y } 6.3 \% \text { respectivamente. } \\
\text { Resulta oportuno mencionar que } \\
\text { estos bajos índices de recaudación } \\
\text { se producen principalmente por un } \\
\text { determinante administrativo al no } \\
\text { desarrollar una adecuada gestión. }\end{array}$ \\
\hline
\end{tabular}




\begin{tabular}{|c|c|c|c|c|c|}
\hline 4. & $\begin{array}{l}\text { Guillermo } \\
\text { y Vargas } \\
\text { (2017) }\end{array}$ & México & $\begin{array}{l}\text { Artículo } \\
\text { científico }\end{array}$ & Descriptivo & $\begin{array}{l}\text { Los gobiernos locales de México } \\
\text { desempeñan una inadecuada gestión } \\
\text { en vista de que el nivel de los índices } \\
\text { de eficiencia obtenidos fue bajo, por } \\
\text { lo que esto ha conllevado la } \\
\text { aparición de irregularidades durante } \\
\text { las actividades al no poder contar } \\
\text { con los fondos requeridos para la } \\
\text { ejecución de obras y proyectos } \\
\text { públicos. Pese a ello, se logró } \\
\text { evidenciar un aumento del importe } \\
\text { recaudado a lo largo de los años, } \\
\text { demostrando así una mejora poco } \\
\text { significativa }\end{array}$ \\
\hline 5. & $\begin{array}{l}\text { Suarez et } \\
\text { al. }(2020)\end{array}$ & Perú & $\begin{array}{l}\text { Artículo } \\
\text { científico }\end{array}$ & $\begin{array}{c}\text { Descriptivo - } \\
\text { no } \\
\text { experimental }\end{array}$ & $\begin{array}{l}\text { La recaudación tributaria por parte } \\
\text { de los municipios presenta diversas } \\
\text { dificultades, generalmente por la } \\
\text { ausencia de recursos necesarios para } \\
\text { la implementación de sistemas y } \\
\text { procesos efectivos, por cuanto el } \\
\text { índice de la brecha que existe por la } \\
\text { ausencia de pago asciende a un } \\
40 \% \text {. Por tanto, se reconoce que la } \\
\text { gestión desarrollada es deficiente } \\
\text { porque no se proporcionan medios } \\
\text { de pago acordes con la realidad } \\
\text { local ni se cuenta con una propuesta } \\
\text { de cultura tributaria que informe a } \\
\text { los contribuyentes sobre asuntos } \\
\text { referidos al tema en cuestión. }\end{array}$ \\
\hline 6. & $\begin{array}{l}\text { Manrique y } \\
\text { Narváez } \\
(2020)\end{array}$ & Perú & $\begin{array}{l}\text { Artículo } \\
\text { científico }\end{array}$ & Explicativo & $\begin{array}{l}\text { Los índices de recaudación } \\
\text { promedio en los departamentos del } \\
\text { Perú ascendieron a } 551.9 \text { y } 1272.5 \\
\text { para los años } 2008 \text { y } 2017 \text {, } \\
\text { demostrando de este modo qué los } \\
\text { municipios mejoraron el desempeño } \\
\text { de sus actividades concernientes al } \\
\text { recaudo de tributos, de los cuales el } \\
85 \% \text { del importe recaudado } \\
\text { corresponde a la región Lima. Por } \\
\text { tanto, queda demostrado la } \\
\text { necesidad de que los municipios } \\
\text { cuenten con un plan de gestión } \\
\text { tributaria que facilite el seguimiento } \\
\text { de acciones eficientes para el } \\
\text { proceso de cobranza. }\end{array}$ \\
\hline 7. & $\begin{array}{c}\text { Gutiérrez } \\
(2020)\end{array}$ & Perú & $\begin{array}{l}\text { Artículo } \\
\text { científico }\end{array}$ & $\begin{array}{c}\text { No } \\
\text { experimental - } \\
\text { correlacional }\end{array}$ & $\begin{array}{l}\text { Se evidencia bajos índices de } \\
\text { recaudación tributaria en la } \\
\text { localidad debido a que no existe } \\
\text { cultura tributaria por parte de los } \\
\text { contribuyentes y el inadecuado }\end{array}$ \\
\hline
\end{tabular}




\begin{tabular}{|c|c|c|c|c|c|}
\hline & & & & & $\begin{array}{l}\text { desarrollo de actividades por parte } \\
\text { de la entidad, y todo ello ha } \\
\text { conllevado a que la gestión } \\
\text { tributaria sea deficiente. Por tal } \\
\text { motivo, frente a la problemática } \\
\text { presentada, las autoridades locales } \\
\text { consideraron conveniente } \\
\text { implementar un sistema tributario } \\
\text { que brinde facilidades para el } \\
\text { seguimiento oportuno de las } \\
\text { acciones de cobranza dirigidas a los } \\
\text { contribuyentes. }\end{array}$ \\
\hline 8. & $\begin{array}{l}\text { Vera } \\
(2019)\end{array}$ & Perú & $\begin{array}{l}\text { Artículo } \\
\text { científico }\end{array}$ & Descriptivo & $\begin{array}{l}\text { El nivel de recaudación tributaria de } \\
\text { la entidad fue bajo en comparación } \\
\text { a los años anteriores debido a que el } \\
\text { importe recaudado no alcanza los } \\
\text { índices proyectados para el presente } \\
\text { año. De igual, forma se evidencia un } \\
\text { incremento en el grado de } \\
\text { morosidad en un } 24.08 \% \text { y } 46.53 \% \\
\text { para el impuesto predial y arbitrios } \\
\text { respectivamente como resultado del } \\
\text { bajo nivel de cultura tributaria y la } \\
\text { falta de voluntad por parte de los } \\
\text { contribuyentes para realizar el pago } \\
\text { respectivo por los tributos } \\
\text { generados, el cual conlleva a que la } \\
\text { entidad presenta dificultades para } \\
\text { poder financiar el desarrollo de } \\
\text { actividades, programas y proyectos } \\
\text { orientados al bienestar social, } \\
\text { siendo este representado por el bajo } \\
\text { nivel de calidad de vida. }\end{array}$ \\
\hline 9. & $\begin{array}{l}\text { Ruiz } \\
\text { (2017) }\end{array}$ & Perú & $\begin{array}{l}\text { Artículo } \\
\text { científico }\end{array}$ & $\begin{array}{c}\text { Correlacional } \\
\text { descriptivo }\end{array}$ & $\begin{array}{l}\text { La gestión municipal se desarrolla } \\
\text { inadecuadamente y ello genera que } \\
\text { no se logre obtener los importes de } \\
\text { recaudación esperados. Del mismo } \\
\text { modo, se puede reconocer que para } \\
\text { el } 2014 \text { solo se logró recaudar } \\
\text { S/7'523,979.60, mientras que el } \\
\text { importe no recaudado ascendió a } \\
\text { S/3'395,261.74, dejando en } \\
\text { evidencia de este modo la existencia } \\
\text { de un índice de morosidad } \\
\text { equivalente a } 30.66 \% \text {. Por tal } \\
\text { motivo la entidad no pudo disponer } \\
\text { de los fondos necesarios para } \\
\text { brindar los servicios públicos de } \\
\text { forma eficiente. }\end{array}$ \\
\hline
\end{tabular}




\begin{tabular}{|c|c|c|c|c|c|}
\hline 10. & $\begin{array}{l}\text { Saavedra y } \\
\text { Delgado } \\
(2018)\end{array}$ & Perú & $\begin{array}{l}\text { Artículo } \\
\text { científico }\end{array}$ & Descriptivo & $\begin{array}{l}\text { En la mayor parte de los estudios } \\
\text { analizados se pudo evidenciar que } \\
\text { existió un bajo nivel de recaudación } \\
\text { tributaria por parte de los gobiernos } \\
\text { locales, siendo esto resultado de la } \\
\text { deficiente gestión tributaria } \\
\text { municipal, ausencia de información } \\
\text { clara y actualizada sobre los } \\
\text { reportes de recaudación de los } \\
\text { periodos anteriores y registro de los } \\
\text { contribuyentes, falta de actitudes } \\
\text { por parte del personal encargado de } \\
\text { la cobranza de tributos, bajo nivel de } \\
\text { cultura tributaria en la población, } \\
\text { etc. }\end{array}$ \\
\hline 11. & $\begin{array}{l}\text { Seminario } \\
\text { y Samamé } \\
(2018)\end{array}$ & Perú & $\begin{array}{l}\text { Artículo } \\
\text { científico }\end{array}$ & $\begin{array}{l}\text { Explicativo - } \\
\text { no } \\
\text { experimental }\end{array}$ & $\begin{array}{l}\text { El nivel de recaudación en el } \\
\text { municipio ha incrementado al año } \\
2017 \text { donde el importe recaudado } \\
\text { por concepto de impuesto predial } \\
\text { fue equivalente a S/3'003,374.00, } \\
\text { mientras que el importe de } \\
\text { morosidad fue S/10'327,117.00, el } \\
\text { cual ha representado un } 5,36 \% \text { del } \\
\text { presupuesto ejecutado. Tales } \\
\text { importes han sido favorables para la } \\
\text { entidad debido a que ha permitido } \\
\text { ejecutar gran parte de los proyectos } \\
\text { programados. De acuerdo con tales } \\
\text { datos, se determina que la adecuada } \\
\text { gestión municipal favorece con la } \\
\text { obtención de ingresos y promueve el } \\
\text { desarrollo de la localidad. }\end{array}$ \\
\hline 12. & $\begin{array}{l}\text { Idrogo } \\
\text { (2018) }\end{array}$ & Perú & $\begin{array}{l}\text { Artículo } \\
\text { científico }\end{array}$ & $\begin{array}{l}\text { Descriptivo - } \\
\text { no } \\
\text { experimental }\end{array}$ & $\begin{array}{l}\text { La deficiente gestión de la } \\
\text { municipalidad produjo como } \\
\text { principal efecto el incumplimiento } \\
\text { de metas presupuestadas elaboradas } \\
\text { para cada año, siendo necesaria la } \\
\text { implantación de medidas o acciones } \\
\text { estratégicas que favorezcan a la } \\
\text { optimización del nivel de } \\
\text { recaudación. Asimismo, se pudo } \\
\text { reconocer que una de las principales } \\
\text { razones que originan la deficiente } \\
\text { gestión es la falta de aptitudes y } \\
\text { buenas prácticas respecto a la } \\
\text { calidad de atención por parte del } \\
\text { personal competente, lo cual genera } \\
\text { que los contribuyentes presenten } \\
\text { dificultades para la adquisición de } \\
\text { información sobre los tributos que } \\
\text { debe cancelar. }\end{array}$ \\
\hline
\end{tabular}


En función a los resultados presentados en la tabla anterior, se reconoce la forma en la que se lleva a cabo la gestión de la recaudación tributaria en las municipalidades del Perú durante los últimos años. En tal sentido, en las investigaciones revisadas se pudo reconocer que los gobiernos locales peruanos no desempeñan una adecuada gestión a causa de diferentes factores, entre las cuales destacan los administrativos; es decir, no se cuenta con talento humano idóneo con las aptitudes y actitudes necesarias para desempeñar sus atribuciones apropiadamente. Asimismo, sobre los factores legales y jurídicos, las municipalidades no cuentan con planes de gestión ni políticas bien definidas sobre los aspectos y procesos que deben ejecutar con el propósito de asegurar el cobro correspondiente de los tributos. Por otra parte, respecto a los factores económicos, destaca la falta de disponibilidad de fondos que puedan ser empleados para la implementación de un sistema o ejecución de un plan de recaudación de modo que se logren las metas presupuestadas. Por último, en lo que refiere a los factores culturales sociales, resalta la ausencia de programas, talleres o presentaciones que proporcionen información a los contribuyentes sobre los principales aspectos que deben tener en cuenta para tributar de forma apropiada, incentivando además al pago responsable de los tributos. Estos resultados concuerdan principalmente con lo referido por Camacho et al. (2017), quienes precisan además sobre lo importante que es para una municipalidad contar con una normatividad tributaria acorde con la realidad que se presenta dentro de cada contexto a fin de que pueda facilitar la reducción de las irregularidades que se presentan durante la ejecución de las actividades de gestión.

De la misma forma, se evidencia que las deficiencias que se presentan durante la ejecución de actividades ocasionan efectos negativos significativos en el desempeño de la municipalidad y el desarrollo social como tal, dado que limita la prestación de servicios públicos de calidad al no poder contar con el dinero necesario para que sea debidamente financiado, impide la cancelación oportuna de los gastos administrativos y operativos que incurre la municipalidad como parte de sus actividades de gestión, imposibilita que el municipio obtenga un desempeño eficiente, entre otros. Estos resultados son similares a los sustentados por Gutiérrez (2020), quien asegura que el principal hecho negativo que suscita el desarrollo deficiente de la gestión de recaudación es la aparición de contingencias o dificultades para poder adquirir el ingreso suficiente para financiar las obras y proyectos públicos cuyo propósito es cubrir ciertas carencias o necesidades de la población. 
Igualmente, se pudo constatar que las municipalidades aplican diversas medidas para optimizar su gestión de recaudación, siendo una de las más usuales la aplicación de censos operativos que permitan contar con la información suficiente para elaborar reportes e informes actualizados que faciliten el control continuo de las actividades; otra de las estrategias aplicables es el dictamen de sanciones más drásticas que permitan frenar el crecimiento acelerado de la cantidad de contribuyentes morosos; indistintamente, otra de las estrategias es la capacitación constante del talento humano para enriquecer sus aptitudes $\mathrm{y}$ actitudes de tal manera que puedan orientar y absolver las dudas que presentan los contribuyentes durante el proceso de pago de sus tributos. Estos resultados son congruentes con la fundamentación de Idrogo (2018), quien dio a conocer que existen una serie de estrategias con gran impacto que pueden ser aplicables dentro de las municipalidades con la finalidad de optimizar las actividades de gestión de recaudación.

Finalmente, en concordancia con las premisas anteriores, se deduce que la gestión de la recaudación en las municipalidades del Perú durante los últimos años fue deficiente por la presencia de una serie de factores limitantes que tuvieron como principal efecto el incumplimiento de las metas de ingresos previstos en el presupuesto para los ejercicios económicos, siendo esencial para ello la aplicación de medidas o acciones estratégicas acordes con la coyuntura que afronta cada municipalidad.

\section{CONCLUSIÓN}

- La gestión de la recaudación en las municipalidades del Perú durante los últimos años se ha agravado y presenta una serie de desatinos en el sistema administrativo; por cuanto, no existen mecanismos efectivos para disciplinar la economía informal, la evasión tributaria y la corrupción. Las actuales medidas represivas no funcionan en contextos donde la evasión y la morosidad predial es masiva.

- Otro problema de la recaudación tributaria que afrontan las municipalidades, es la opinión confusa y negativa que tienen los contribuyentes y la población en general sobre el uso del dinero que se recauda como consecuencia de algunas malas prácticas de gestión municipal; a esto se suma, el desconocimiento de los ciudadanos sobre la importancia de la tributación para el desarrollo. En efecto, se requiere fomentar conocimiento y hábitos tributarios en los contribuyentes mediante el empleo de políticas más pertinentes y sanciones positivas que incentive y concienticen sobre la ventaja de ser formales frente a la informalidad. 
- La poca difusión sobre el uso adecuado de los recursos recaudados, la escuálida conciencia y cultura tributaria en los contribuyentes más la ineficiencia administrativa de las municipalidades del Perú frente a la informalidad, evasión y corrupción del sistema, entre otros factores directos e indirectos que ocasionan la baja recaudación; son razones suficientes para plantear una propuesta de gestión más efectiva o un nuevo sistema tributario acorde con la realidad de cada municipio.

\section{REFERENCIAS}

Camacho, C., Hurtado, K., Navarro, E., Hurtado, J. y Nieves, S. (2017). Factores que inciden en el recaudo del Impuesto Predial Unificado en el distrito de Barranquilla. Prospectiva, 15(1),

64-73. http://ojs.uac.edu.co/index.php/prospectiva/article/view/591

Chávez, L. y López, V. (2019). Determinantes del recaudo del impuesto a la propiedad inmobiliaria: un enfoque estadístico para México. Revista Iberoamericana de Estudios Municipales, (19), 89-119. http://dx.doi.org/10.4067/S071917902019000100089

Díaz-Narváez, V. y Calzadilla, A. (2016). Artículos científicos, tipos de investigación y productividad científica en las Ciencias de la Salud. Revista Ciencias de la Salud, 14(1), 115-121. https://www.redalyc.org/pdf/562/56243931011.pdf

Gonzáles, A. y Mosquera, L. (2020). Del control previo y perceptivo al posterior y preventivo: estudio de la trayectoria en el control fiscal en Colombia (1991-2019). Revista Virtual Via Inveniendi et Iudicandi, 15(1), 71-90. https://doi.org/10.15332/19090528/5742

Guillermo, S. y Vargas, I. (2017). Recaudación potencial, eficiencia recaudatoria y transferencias federales: Un análisis para las entidades federativas en México utilizando el modelo de frontera estocástica. EconoQuantum, 14(1), 35-71. http://www.scielo.org.mx/scielo.php?script=sci_abstract\&pid=S1870$66222017000100035 \& \operatorname{lng}=\mathrm{es} \& \mathrm{nrm}=$ iso

Gutiérrez, J. (2020). Gestión de administración tributaria y su influencia en la recaudación del impuesto predial Municipalidad Distrital Jose Leonardo Ortiz, Chiclayo 2018. Revista Ciencia y Tecnología, 16(2), 165-176. https://revistas.unitru.edu.pe/index.php/PGM/article/view/2911 
Idrogo, M. (2018). Estrategias para mejorar la recaudación tributaria en la Municipalidad Provincial de Chota. Revista Ciencia Nor@ndina, 1(2), 104-111. http://unach.edu.pe/rcnorandina/index.php/ciencianorandina/article/view/108/73

Manrique, J. y Narváez, J. (2020). Niveles de recaudación tributaria e inversión pública a nivel departamental en el Perú, 2008 - 2017. Revista Ciencia UNEMI, 13(33), 108-119. https://doi.org/10.29076/issn.2528-7737vol13iss33.2020pp108-119p

Melgarejo, V. (2018, 2 de noviembre). Municipalidad de Lima enfrenta su mayor caída en recaudación de impuestos municipales. Diario Gestión. https://n9.cl/njh6

Ministerio de Economía y Finanzas (2019). Diagnóstico del Impuesto Predial en el Perú. Dirección General de Política de Ingresos Públicos. https://n9.cl/iz9uv

Morel, J. Trivelli, C., Vásquez, Y. y Mendoza, J. (2020). Poder y tributación en el Perú: Un balance bibliográfico. Instituto de Estudios Peruanos. https://n9.cl/mjk3d

Onofre, R., Aguirre, C. y Murillo, K. (2017). La cultura tributaria y su incidencia en la recaudación de los tributos en el Cantón Babahoyo, Provincia de los Ríos. Dominio de las $\quad$ Ciencias, 3(3), 3-23. https://dialnet.unirioja.es/servlet/articulo?codigo $=6326643$

Pardal-Refoyo, J. y Pardal-Peláez, B. (2020). Anotaciones para estructurar una revisión sistemática. Revista $\quad$ ORL, 11(2), 155-160. https://scielo.isciii.es/scielo.php?script=sci_arttext\&pid=S244479862020000200005

Pérez, C., Espinosa, I. y Londoño, H. (2019). Las cuentas claras. Ingresos fiscales en las principales ciudades colombianas. Cámara de Comercio de Cali. https://www.ccc.org.co/inc/uploads/2019/04/Compas_03_LasCuentasClaras.pdf

Redacción Andina (2020, 28 de abril). MEF transferirá en mayo S/ 800 millones a municipios por cumplir metas del 2019. Diario Andina. https://n9.cl/c0zt

Ruiz, J. (2017). La cultura tributaria y la gestión municipal. Quipukamayoc, 25(48), 4960. http://dx.doi.org/10.15381/quipu.v25i48.13992

Saavedra, R. y Delgado, J. (2020). La recaudación tributaria municipal 2020. Revista $\begin{array}{llll}\text { Multidisciplinar } \quad \text { Ciencia } & \text { 2atina, }\end{array}$ https://doi.org/10.37811/cl_rcm.v4i2.109 
Sánchez, F. (2019). Fundamentos epistémicos de la investigación cualitativa y cuantitativa: consensos y disensos. Revista Digital de Investigación en Docencia Universitaria, 13(1), 102-122. http://dx.doi.org/10.19083/ridu.2019.644

Seminario, F. y Samamé, S. (2018). Recaudación tributaria y su incidencia en el desarrollo local de la Provincia de Moyobamba en el período 2010-2018. Revista Gobierno $\quad y \quad$ Gestión Pública, 5(1), 73-90. https://doi.org/10.24265/iggp.2018.v5n1.05

Suarez, H., Palomino, G. y Aguilar, C. (2020). Gestión de recaudación tributaria municipal: Una visión cultural. Ciencia Latina Revista Científica Multidisciplinar, 4(2), 635-654. https://doi.org/10.37811/cl_rcm.v4i2.105

Vera, P. (2019). Influencia de la fiscalización en la recaudación tributaria de la Municipalidad Distrital de Víctor Larco de Trujillo. Sciéndo, 22(2), 93-98. https://revistas.unitru.edu.pe/index.php/SCIENDO/article/view/2400/pdf 